\title{
Treatment Techniques in External Beam Radiation Therapy: A Review
}

\author{
Sant Pal Singh ${ }^{1 *}$, Asita Kulshreshtha ${ }^{1 *}$, Anoop Kumar Srivastava ${ }^{2}$ \& Sumit Kumar Srivastava ${ }^{1}$ \\ ${ }^{1}$ Amity School of Applied Sciences, Amity University Uttar Pradesh, Lucknow \\ ${ }^{2}$ Dept. of Radiation Oncology, Dr. RML Institute of Medical Sciences, Lucknow \\ DOI: 10.29322/IJSRP.11.09.2021.p11706 \\ http://dx.doi.org/10.29322/IJSRP.11.09.2021.p11706
}

\begin{abstract}
Radiation therapy being most general treatment modality to cure patients with different malignancies covers more than fifty percent of cancer burden. Since its inception for treatment of cancer and other malignancies in 1896, development of new devices from time to time has brought several changes in techniques of radiation therapy. The present study aims to present an outlook towards the basic concepts involved related to radiation therapy and to highlight the development of the various techniques of radiation therapy involving wide range of energies from x-ray, $\gamma$-ray and charged particles. This presentation gives an outlook for various advanced techniques of radiation therapy like external beam radiation therapy, intensity modulated radiation therapy, image guided radiation therapy, stereotactic radiation therapy, brachy-therapy etc. This study gives an introduction ofutilization of x-rays, $\gamma$-rays, photon beams and light charged particles i.e. electrons as a source of radiation used for radiation therapy andit is concluded that the electron beam therapy is more suitable for superficial tumors like nasal nodes, head and neck cancers and breast carcinomas. It is also beneficial in providing the radiation boost to the residual cancers after surgery. Since the energy of the beam drop off rapidly after the desired depth, so that the sensitive body parts can be escaped from the radiation effects.
\end{abstract}

Index Terms- Electromagnetic radiation, brachytherapy, computed tomography, radiation boost, biomedical imaging, on board imaging and multileaf collimators etc.

\section{INTRODUCTION}

$\mathrm{U}$ tilization of ionizing radiations were initiated totreatment of cancer and other malignancies just after the introduction of X-rays (a pattern of electromagnetic radiation) by German Professor Wilhelm Conrad Röntgen in 1895 and detection of radium by Marie Curie 1898[1,2]. Radiobiological properties of Xrays and radium were recognized just after their evolution.Within a year of diagnosis of x-rays, Emil Herman Grubbe of Chicago assembled his first $\mathrm{x}$-ray machine and used it fortreatment of breast carcinomain 1896 [3].First conference concerned with contribution of radiation physics in medicine was organized in 1913.In early days of radiation therapy, x-rays and radium were only source of radiation for therapeutic cure of cancer as well as other malignancies and achieving around $20 \%$ of curable treatment [4]. Radiation physicists dealing with x-rays and gamma rays faced skin burns and many matured non-healing ulcers that predict towards biological properties of these radiations [5].
Diagnostic and therapeutic utilizations of radiation led to revolutionary changes in the field of medical science. Depending on radiobiological properties of radiations they are used in treatment of cancers, non-healing ulcers and other skin diseases [6-8].H. Strebel from Germany in 1903 and R. Abbe from New York in 1904 respectively were the first, who used these radiations in interstitial brachytherapy $[9,10]$. Cancers of cervix was successfully treated with breachy-therapy in 1913 at congress organized at Halle. Breachytherapy was only technique for treatment of most of the patients suffering with cancer of cervix [11]. Three Physicists, for their discoveries related to radiations received Nobel Prize in late nineteenth century as golden era of Physics.Several other developments in this field took place such as production of isotopes, radiobiology and the three dimensional (3D) dosimetry [12-18]. Successive development of computed tomography (CT), magnetic resonance imaging (MRI), positron emission tomography (PET) and other advancedimaging techniques led to dosage prescriptionand treatment plan from print to volume and also single plane dosimetry to multiple planedosimetry [19]. Discoveriesof basic radiation sciences (i.e. $\mathrm{x}$-rays and $\gamma$-rays) direct towards keen understanding of human body disorders and treatments techniques lead to revolutionary development in medical sciences and diseases managements [2023].

\section{METHODS AND MATERIALS}

\subsection{Computed Tomography and its Development}

A revolutionary improvement took place in radio-diagnosis as well asradiation therapy planning of cancer with the development of advanced imaging techniques like CT (figure 1), MRI and PET-CT. Initially, it was very difficult to determine radiation dose distribution in different sites and organs of human body for irradiation. Isodose charts obtained from homogeneous medium (water equivalent) were the only basis of radiation dose calculations [24,25]. However, there are many distortions due to inhomogeneities of human body and hence estimation of exact dose of radiation inside body was not possible. The exact absorption pattern, electron fluence and point to point dose corrections were needed to plan accurate radiation therapy. It was not possible with the conventional treatment techniques so that for deep rooted tumors one has to guess relative position of tumor and normal tissues.

Utility of x-rays were redeemed with emergence of CT in 1971 by Dr. G.N. Hounsfield and EMI Ltd. They developed the first commercial system capable of image reconstruction from $\mathrm{x}$ - 
rays projections in 1972 [26]. These systems were trialed with $\gamma$ rays source in experimental mode and later on replaced by x-rayssource. Ledly in 1974 developed the first whole body scanner with ameliorated spatial resolution which was used for CT supported treatment planning [27].

Later on advanced mathematical principles were used in image reconstruction algorithms to have higher resolutions. These CT scanners can scan pictures in few seconds or less with higher resolution and sensitivity. Advance CT treatment plannings improve outcomes of radiation therapy such as various inhomogeneities inside body like air cavities (lungs, oral cavity, nasal cavity etc.), bones and tissues of different densities and composition. More quantitative and precise information regarding patient cross-section, shape and composition (effective atomic number, density etc.) of inhomogeneities are available with advanced CT scanners [28].Various stages of the developments in computed tomography is given below-

2.2.1 First generation:First generation of computed tomography is based on the concept of rotate/translate system of pencil beam of x-rays. Pencil beam of $\mathrm{x}$-rays was produced by using a pinhole collimator for producing a single beam of $\mathrm{X}$-rays to interact with the organ. First generation computed tomography scanners were able to measure the amount of $x$-rays passing through the organ successfully by using only two detectors located on the opposite sides of the body organ. Time taken for imaging and their reconstruction was major drawback of first generation computed tomography scanners [29].

2.2.2 Second generation:Second generation of computed tomography scanners was introduced with a narrow fan $\mathrm{x}$-rays beam with an angle of $10^{\circ}$ approximately in the form of a linear array of 30 detectors. The largest advantage associated with second generation scanners was the substantial decrease in time in comparison to first generation. Unlike pencil beam, narrow fan beams contribute to scattering and hence detectors were exposed to more scattered beams resulting in decreased resolution of images. Similar to first generation CT scanners, second generation $\mathrm{CT}$ scanners also require the rotation and translation of $\mathrm{x}$-ray beams and hence capable for imaging of head only [30].

2.2.3 Third generation:Further to second generation usage of wide $\mathrm{x}$-rays fan beam of angular range between $40^{\circ}$ to $60^{\circ}$ reduced the consumption of imaging time to 5-20 seconds in third generation of computed tomography scanners. Detectors were in the form of an array of 400-1000 detecting elements. A wide x-ray fan beam and detectors were joined so that they can rotate synchronously to produce faster imaging. Dueto presence of a large number of detectors and uncalibrated detectors third generation CT scanners produce a ring artifact [31-32].

2.2.4 Fourth generation:Fourth generation of computed tomography scanners had around 5000 stationary detectors with perfect synchronization and calibration, arranged in the ring of $360^{\circ}$. Images from these scanners were reconstructed by $\mathrm{x}$-rays fan beams obtained from the rotating $\mathrm{x}$ rays tube. Due to this feature of the set-up, fourth generation CT scanners were specified as rotatestationary geometry [33-35].

2.2.5 Fifth generation: Specially for cardiac tomography fifth generation of computed tomography scanners also known as cine-CT scanners or electron beam scanners were designed with no moving parts and referred to as stationary/stationary geometry. In fifth generation CT scanners the patient lies in the target of a large x-rays tube and electron beam after deflection produces $\mathrm{x}$-rays which were detected by detectors. These scanners were able to capture unique contractions and relaxations of heart with a very high speed of imaging acquisition of the order of $50 \mathrm{msec}$ [36].

2.2.6 Sixth generation:To overcome the gantry related difficulties of previous generation in 1990s sixth generation of computed tomography scanners with helical geometry introducing the slip ring technology for imaging were designed. This generation was the combination of the principles of third and fourth generation. The acquisition time for this generation was very small (i.e. the entire abdomen scanning took around 30 seconds). The data provided by these CT scanners in helical form was the measure drawback of this generation [37].

2.2.7 Seventh generation:Seventh generationof computed tomography scanners is the most recent with cone shaped $\mathrm{X}$-rays beam and multiple detectors. The linear detector array of the previous generation is modified to flat panel detector or to multiple detectors array [38]. Imaging speed and their reconstruction are very outstanding with the combination of cone shaped $\mathrm{x}$-rays beam and paneled detectors, which can acquire very large number of slices in very short time period. 


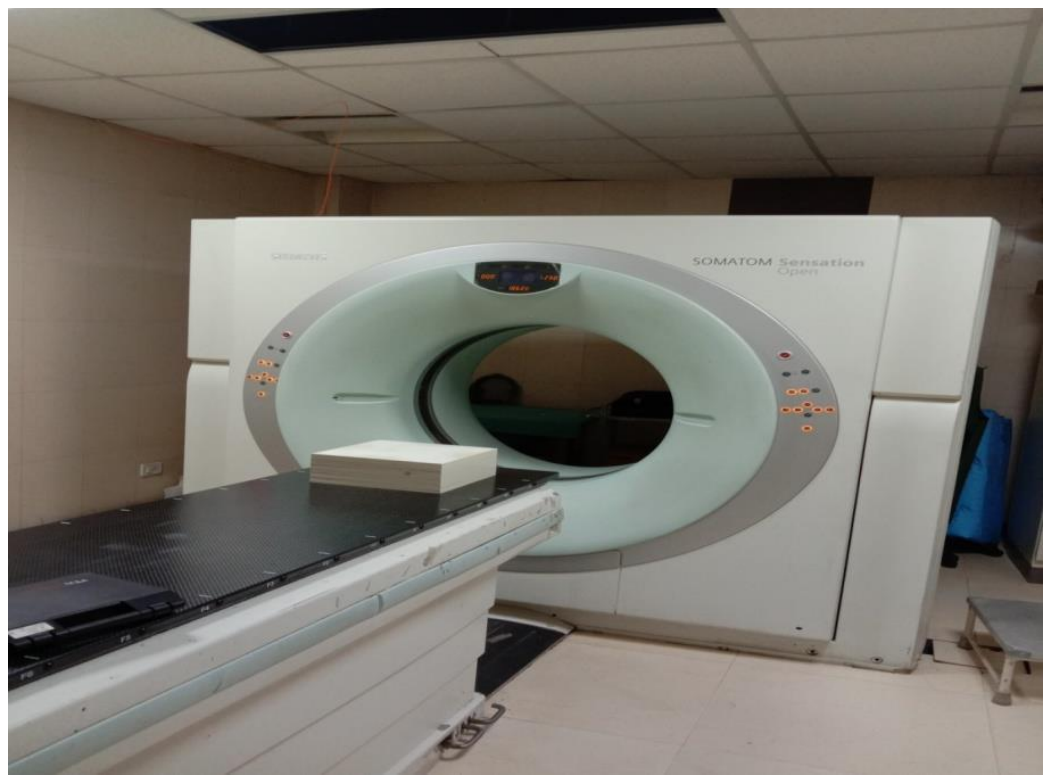

Figure1.Siemens-Somatom Sensation open CTScanner at Dr. Ram Manohar Lohia Institute of Medical Sciences Lucknow, Uttar Pradesh, India

\subsection{Advanced Techniques used inExternal Beam RadiationTherapy}

Radiation delivering technologies and dose escalations for target volume in external beam radiation therapy hadimproved with time. Megavoltage external beam radiation therapy is usually delivered by Cobalt units (isotope $\mathrm{Co}^{60}$, atomic number 27, mass number 60 , mean energy $1.25 \mathrm{MeV}$, half life 5.26 years and $\gamma$-ray constant 1.33 Rontgen/hour-currie at 1 meter distance) in regular practices as the main stay in a radiation source [39]. Selection of beam energy for available technology depending on the depth of tumor has become a standard practice to deliver tumoricidal dose. High energy clinical linear accelerators with On-Board-Imaging (OBI) and computer controlled multileaf collimating (MLC) systems(as shown in figure 2) has escorted prototype changes in radiation therapy practices. It makes possible to deliver the escalated dose to targeted volume and minimized dose within tolerance limits to normal tissues. These radiation techniques deliver highly conformal and homogeneous radiation dose to target with positional accuracy less than $1 \mathrm{~mm}$.

In general a linear accelerator can provide 2-3 photon energies and 4-7 electron energies. The dual energy Clinical Linear accelerators offer an option to select photon energy depending on the depth of tumor. The superficial tumors are treated by low energy photon beam with small penetration depth and the deep seated tumors are treated by high energy photon beam with large penetration depth [40-42]. The electron beam has very small penetration power and found to be very useful for treating the superficial tumor upto $6 \mathrm{~cm}$ depth. Most useful range of energy for electron beams is $6-15 \mathrm{MeV}$. 


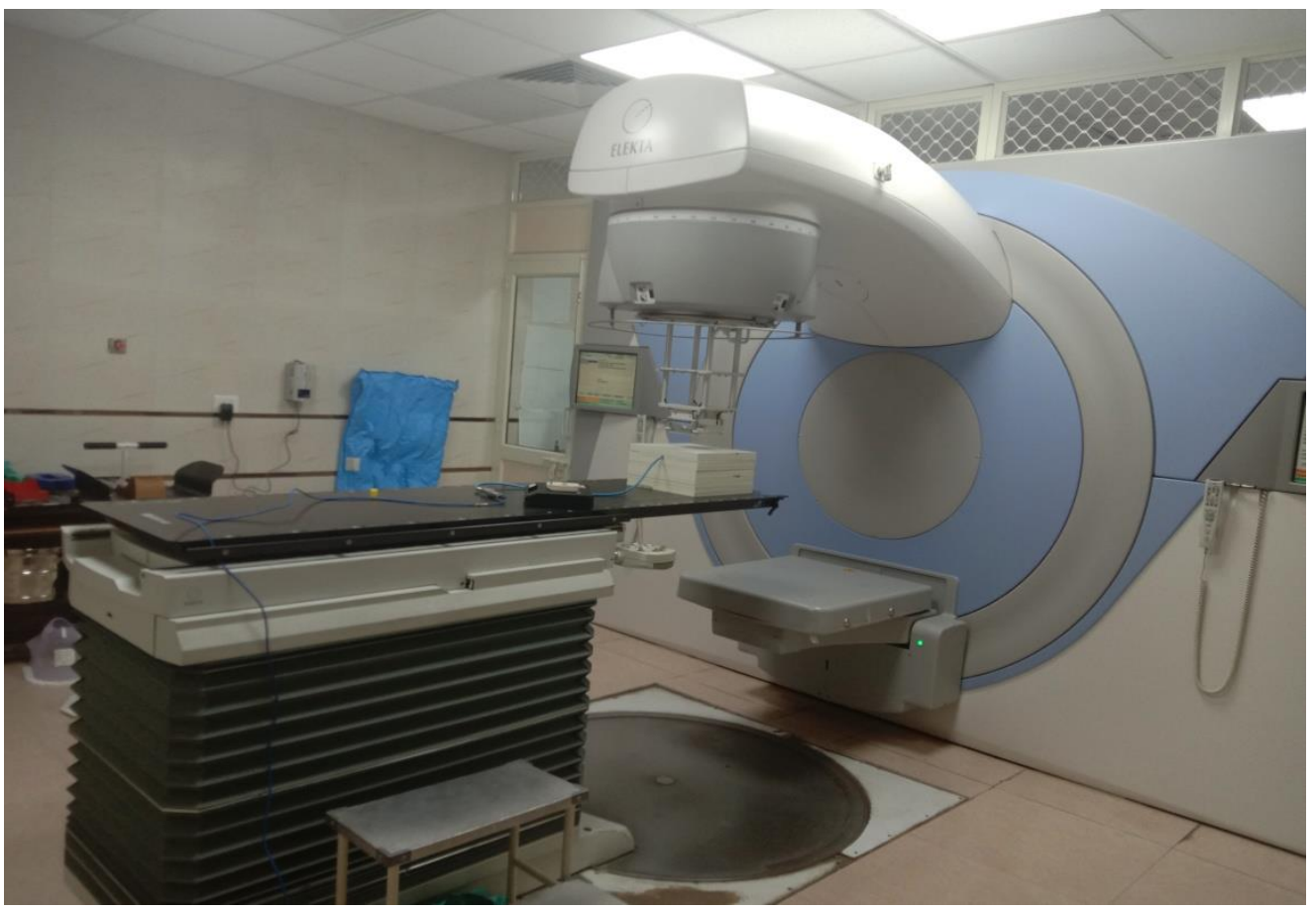

Figure 2. Clinical linear ascelerator "Elekta Synergy" with Multileaf collimator at Dr. Ram Manohar Lohia Institute of Medical Sciences, Lucknow, Uttar Pradesh, India

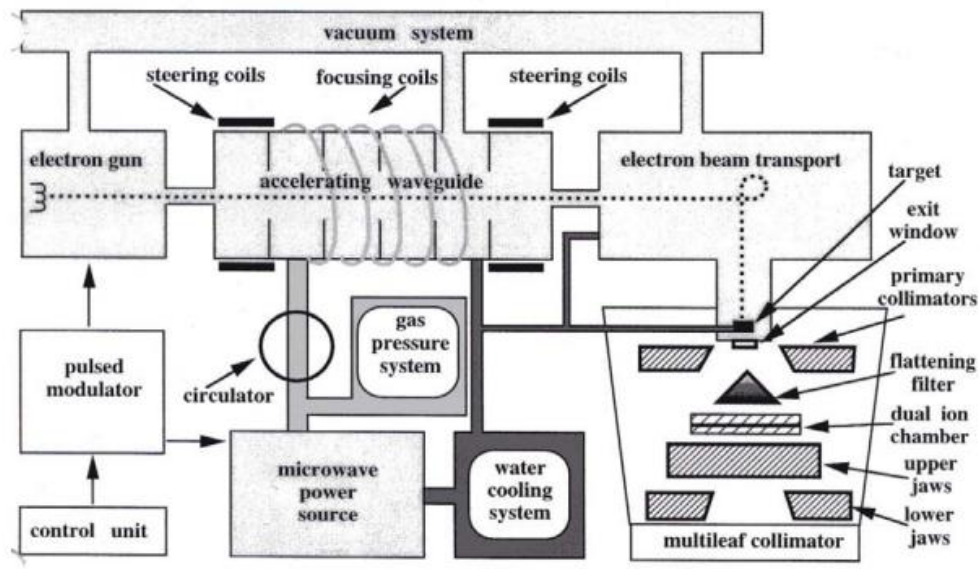

Figure 3. Schematic diagram of Clinical Linear Accelerator with multileaf collimator.

However, in some cases photon beams are used in combination of electron beam to boost superficial nodes after the completion of photon beam treatment. The electron beams are frequently used for irradiation of chest wall carcinoma of breast and to boost the nodes in head and neck tumors. Table 1 below is an outlook to provide information about the $\mathrm{x}$-rays and electron energies available from Clinical Linac in present time [42].

Table 1. Photon and Electron energies received from a Clinical Linear Accelerator: An experimental data showing maximum depth dose for photon beam and $90 \% 80 \%$ dose depth for electron beam.

\begin{tabular}{|l|l|l|l|l|l|l|}
\hline \multirow{2}{*}{ Sl.No. } & Photon & Electron & \multicolumn{3}{|l|}{} \\
\cline { 2 - 6 } & Energy (MV) & $\begin{array}{l}\text { Max. Dose Depth } \\
(\mathrm{cm})\end{array}$ & $\begin{array}{l}\text { Energy(Me } \\
\text { V) }\end{array}$ & $\begin{array}{l}90 \% \text { Dose Depth } \\
(\mathrm{cm})\end{array}$ & $\begin{array}{l}80 \% \text { Dose Depth } \\
(\mathrm{cm})\end{array}$ \\
\hline 1. & 4 & 1.0 & 4 & 1.1 & 1.3 \\
\hline 2. & 6 & 1.6 & 6 & 1.5 & 2.0 \\
\hline 3. & 10 & 2.5 & 9 & 2.2 & 3.0 \\
\hline
\end{tabular}

This publication is licensed under Creative Commons Attribution CC BY. 


\begin{tabular}{|l|l|l|l|l|l|}
\hline 4. & 15 & 2.8 & 12 & 3.0 & 4.0 \\
\hline 5. & 18 & 3.5 & 15 & 3.7 & 5.0 \\
\hline 6. & 24 & 4.0 & 18 & 4.5 & 6.0 \\
\hline 7. & -- & ------ & 21 & 5.2 & 7.0 \\
\hline
\end{tabular}

\subsection{TreatmentPlanningSystem (TPS)in Advanced External Beam Radiation Therapy}

Treatment planning system (TPS) being the backbone of radiation therapy system is a key to improve the outcomes of radiation therapy. TPS provides various treatment options in very efficient manner for radiation therapy. Its outstanding features not only reduce pre treatment planning time but also yield alternatives like self image fusion and fast organ delineation [43-45]. Various advanced treatment techniques to define the best treatment plan are contributed by TPS systems. TPS reinforce are wide range of treatment techniques including two dimensional (2D), three dimensional (3D), MLC based IMRT, SRS, SRT etc. Its advanced tools ease the contouring delineation \& field setup and its high speed calculation engine minimize the typical planning time. The high resolution and less delivery time of TPS reduce the delivery time of advanced treatment modalities to 10-15 minutes o a Clinac with advanced TPS. The option for pal verification and quality assurance provided by TPS systems help Physicists to save time and increase accuracy of the system [46].

TPS systems came into existence in 1980s when one dimensional planning was done manually. 2D-CT based TPS was developed during 1980-1990s with the involvement of computers. After 1990s processes of dose calculations inside inhomogeneous human tissues were enhanced with development of different algorithms like pencil-beam, convolution, Clarkson, FFT Convolution, Multi Grid Superposition, Electron Monte Carlo and Fast Superposition etc. 3D dose calculations came into existence by 1995s and 4D dose calculations with increased outcomes of radiation therapy and real time motion of patient during the treatment were developed upto 2000 [47]. Data sets of CT imaging of the target are directly loaded to the computer system for processing and then TPS system creates a typical complex treatment plan for each beam line and directs the system to deliver the therapy. Computer software calculates the desired dose distribution for the various inhomogeneities of the patient body like bones, lungs, muscles etc.

Involvement of TPS is very fruitful in maximizing the dose to tumor site as well as escaping the critical structures from radiation effects to avoid their damage complications. TPS includes all the beam arrangement and automatic beam modification devices to modify the beam around critical structures.Automated complex programming of TPS for multileaf-collimators is capable to block the critical structures during the treatment delivery [48]. Beam modification facility provided by TPS can modify the beam in case shrinkage of tumor during the treatment time. XiO, Eclipse, Pinnacle, Oncentra, Prowess etc. are various TPS systems used in present time. TPS also gives a choice to choose appropriate algorithm for treatment depending on requirement of case [49].

\section{CONCLUSION}

The present study gives an outlook towards the basic concepts involved related to radiation therapy. This study also gives a brief idea of the development of the various techniques of radiation therapy involve wide range of energies from $\mathrm{x}$-ray, $\gamma$-ray and charged particles. By using this study it is concluded that electron beam external radiation therapy is more suitable for superficial tumors like nasal nodes, head and neck cancers and breast carcinomas. It is also beneficial in providing the radiation boost to the residual cancers after surgery. Since the energy of the beam drop off rapidly after the desired depth, so that the sensitive body parts can be escaped from the radiation effects.

\section{ACKNOWLEDGEMENT}

The author acknowledges that this article has prepared under the supervision of Professor Asita Kulshreshtha, head of Amity school of applied sciences, Amity University Uttar Pradesh, Lucknow and Dr. Anoop Kumar Srivastava, Associate Professor, Department of radiation oncology, Dr. Ram Manohar Lohia Institute of Medical Sciences, Lucknow, Uttar Pradesh.

\section{REFERENCES}

[1] W.C. Röntgen, "Über Eine Neue Art Von Strahlen. Vorlaufige Mitteilung", Sitzungsberichte Der Physikalisch-Medizinischen Gesellschaft $\mathrm{Zu}$ Würzburg, Sitzung. Vol. 30, (1895), pp. 132-141.

[2] M. Lederman, "The early history of radiotherapy, 1895-1939", International Journal of Radiation Oncology, Biology and Physics. Vol. 7, (1981), pp. 639648.

[3] A.H. Becquerel, "Sur les radiations emises par phosphorescence", Comptes Rendus Academic Science. Vol. 122, (1896), pp. 420-421.

[4] P. Curie and M. Curie, "Sur une substance nouvelle radioactive, contenuedans lapechblende", Comptes Rendus Academic Science. (1898), pp. 127-175.

[5] E.H. Grubbe, "Priority in the therapeutic use of X-rays", Radiology. Vol. 21, (1933), pp. 156-162.

[6] W.D. Coolidge, "A powerful Röntgen ray tube with pure electron discharge", Physics Review. Vol. 2, (1913), pp. 409-413.

[7] H.S. Kaplan, "Basic principles in radiation oncology", Cancer. Vol. 39, (1977), pp.689-693

[8] C. Regaud and R Ferroux, "Discordance des effets de rayons X, d'une part dans lapeau, d'autrepartdans le testicule, par le fractionnement de la dose", Comptes Rendus Society of Biology. Vol. 4, (1927), pp. 97-143.

[9] H. Strebel, "Vorschläge zur Radiumtherapie", Deutsche Medizinal-Zeitung Vol. 24, (1903), pp. 1145-1146.

[10] T. Abbe, "Notes on the physiologic and the therapeutic action of radium", Washington Medical Annals. Vol. 2, (1904), pp. 363-376.

[11] R.F. Robison, "The race for megavoltage", Acta Oncol. Vol. 34, (1995), pp. 1055-1074.

[12] W.D. Coolidge, "Cathode-ray and Roentgen-ray work in progress", Am Journal Roentgenol. Vol. 19, (1928), pp. 313-321.

[13] E.D. Courant, "Early Milestones in the Evolution of Accelerators", Reviews of Accelerator Science and Technology. Vol. 1, ed. by A.W. Chao, World Scientific, Singapore. (2008).

[14] R.J. Van de Graaff, "A 1500000 volt electrostatic generator", Physics Review. Vol. 38, (1931), pp.1919-1920.

[15] J.D. Cockcroft, "Walton ETS. Experiments with high velocity positive ions", Proc R Soc Lond A. Vol. 129, (1930), pp. 477-489.

[16] C.D. Cockcroft and E.T.S. Walton, "Experiments with high velocity positive ions, and Further developments in the method of obtaining high velocity positive ions", Proc R Soc Lond A. Vol. 136, (1932), pp. 619-630. 
[17] E.O. Lawrence and M.S. Livingston, "The production of high speed light ions without the use of high voltages", Physics Review. Vol. 40, (1932), pp. 1935

[18] R.S Stone, J.H. Lawrence and P.D. Aebersold, "A preliminary report on the use of fast neutrons in the treatment of malignant disease", Radiology. Vol. 37, (1940), pp. 322-327.

[19] D.W. Kerst, "The Acceleration of electrons by magnetic induction", Physics Review. Vol. 8, (1940), pp. 841

[20] D.W. Kerst, "The acceleration of electrons by magnetic induction", Physics Review. Vol. 60, (1941), pp. 47-53.

[21] C.J. Karzmark and N.C. Pering, "Electron linear accelerators for radiation therapy from history, principles, and contemporary developments", Physics Med Biol. Vol. 18, (1973), pp.321-354.

[22] R.J. Berry, "Therapeutic uses of X-rays", International Journal of Radiation Biology. Vol. 15, (1985), pp. 873-895.

[23] H.S. Kaplan, "Historic milestones in radiobiology and radiation therapy", Semin Oncology. Vol. 6, (1979), pp. 479-489.

[24] M. Tubiana, "Comment on Computed Tomography and Radiation Exposure", N. Engl. J. Med. Vol. 358, no. 8, (2008), pp. 852-853.

[25] Jun, Kyungtaek, Yoon and Seokhwan, "Alignment Solution for CT Image Reconstruction using Fixed Point and Virtual Rotation Axis", Scientific Reports. Vol. 7, (2017), pp. 41218.

[26] G.N. Hounsfield, "Computerized transverse axial scanning in Description of the system" Br Journal Radiology. Vol. 46, (1973), pp. 1016-1022.

[27] J. Ambrose, "Computerized transverse axial scanning in second Clinical application”, Br Journal Radiology. Vol. 46, (1973), pp. 1023- 1047

[28] F.M. Khan, "The physics of radiation therapy, 4th edition", Philadelphia: Lippincott Williams and Wilkins. (2010).

[29] J. Hsieh, "CT Image J. Hsieh, "CT Image Reconstruction, in RSNA Reconstruction, in RSNA Categorical Course in Categorical Course in Diagnostic", Diagnostic Radiology Physics, CT and US Radiology Physics, CT and US Cross -sectional Imaging 2000 sectional Imaging 2000, ed. L. W. Goldman, J. B. L. W. Goldman and J. B. Fowlkes, RSNA, (2000), pp. 53 64.

[30] C.M. Washington and D.T. Leaver, "Principles and Practice of Radiation Therapy", Physics, Simulation, and Treatment Planning, 1st ed. Maryland Heights Mosby. (2003).

[31] E.C. Beckmann, "CT scanning in the early days", The British Journal of Radiology. Vol. 79, no. 937,(2006), pp. 5-8

[32] V.K. Narayanan, R. Vaitheeswaran, J.R. Bhangle, S. Basu, V. Maiya and B. Zade, "An experimental investigation on the effect of beam angle optimization on the reduction of beam numbers in IMRT of head and neck tumors", Journal of Applied Clinical Medical Physics. Vol. 13, (2012), pp. 3912.

[33] Z.V. Maizlin and P.M. Vos, "Do we really need to thank the Beatles for the financing of the development of the computed tomography scanner", Journal of Computer Assisted Tomography. Vol.36, no. 2, (2012), pp. 161-164.

[34] K.S. Clifford Chao, S. Apisarnthanarax, and G. Ozyigit, "Practical Essentials of Intensity Modulated Radiation Therapy", 2nd ed. Philadelphia Lippincott Williams and Wilkins. (2005).

[35] O.P. Gurjar and S.P. Mishra, "Dosimetric analysis of intensity modulated radiotherapy plans having one or more pairs of parallel opposed beams among the set of beams in some special cases", Radiation Proton Environ. Vol. 36, (2013), pp. 138-142.

[36] T.G. Flohr, K. Stierstorfer and S. Ulzheimer, "Image reconstruction and image quality evaluation for a 64-slice CT scanner with z-flying focal spot", Medical Physics. Vol.32, no. 8, (2005), pp. 2536-2547.

[37] A.W. Leber, A. Knez, F. Von Ziegler, A. Becker, K. Nikolaou, S. Paul, B. Wintersperger, M. Reiser, C.R. Becker, G. Steinbeck, and P. Boekstegers, "Quantification of obstructive and non obstructive coronary lesions by 64 - slice computed tomography", Journal ACC. Vol. 46, no. 1, (2005), pp. 147154.

[38] M. Mori, M. Endo, T. Tsunoo, S. Kandatsu, S. Tanada and H. Aradate, "Physical performance evaluation of a 256-slice CT-scanner for fourdimensional imaging", Medical Physics. Vol. 31, no. 6 (2004), pp. 13481356.

[39] J. Lopez-Tarjuelo, R. Garcia-Molla, X.J. Juan-Senabre, J.D. QuirosHigueras, A. Santos-Serra and N. de Marco-Blancas, "Acceptance and commissioning of a treatment planning system based on Monte Carlo calculations", Technol Cancer Res Treat. Vol. 13, (2014), pp. 129-138.

[40] J. Ojala, "The accuracy of the Acuros XB algorithm in external beam radiotherapy-a comprehensive review", International Journal of Cancer Therapy Oncology. Vol. 2, (2014), pp. 20417.

[41] N. Hodapp, "The ICRU Report 83, Prescribing, recording, and reporting photon beam intensity-modulated radiation therapy", Strahlenther Oncology. Vol. 188. (2012), pp. 97-99.

[42] J.E. Marks, A.G. Haus, H.G. Sutton and M.L. Griem, "The value of frequent treatment verification films in reducing localization error in the irradiation of complex fields", Cancer. Vol. 37, (1976), pp. 2755-2761.

[43] J. Bijhold, K.G. Gilhuijs and M. Van Herk, "Automatic verification of radiation field shape using digital portal images", Medical Physics. Vol. 19, (1992), pp. 1007-1014.

[44] O.P. Gurjar, S.P. Mishra, V. Bhandari, P. Pathak, P. Pant and P. Patel, “A study on the necessity of $\mathrm{kV}$-CBCT imaging compared to $\mathrm{kV}$-Orthogonal portal imaging based on setup errors: Considering other socio-economical factors", Journal of Cancer Research Therapy. Vol. 10, (2014), pp. 583-586.

[45] Y.Z. Wang, M.D. Evans and E.B. Podgorsak, "Characteristics of induced activity from medical linear accelerators", Medical Physics. Vol. 32, (2005), pp. 2899-2910.

[46] N. Ebert, F. Tillner and M. Baumann, "Encyclopedia of Cancer (Third Edition)", Radiation Oncology. (2019), pp. 321-336.

[47] Fraass, A. Benedick (1995). "The development of conformal radiation therapy". Medical Physics. Vol. 22, no. 11, (1995), pp. 1911-1921.

[48] A. Karabis, P. Belloti, D. Baltas, O. Dössel and W.C. Schlegel (eds.), "Optimization of Catheter Position and Dwell Time in Prostate HDR Brachytherapy using HIPO and Linear Programming", World Congress on Medical Physics and Biomedical Engineering. IFMBE Proceedings. Vol. 25, no. 1, (2009), pp. 612-615.

[49] D. Gintz, K. Latifi, J. Caudell, B. Nelms, G. Zhang, E. Moros, and V. Feygelman, "Initial evaluation of automated treatment planning software", Journal of Applied Clinical Medical Physics. Vol. 17, no. 3, (2016), pp. 331346.

\section{AUTHORS}

First Author - Sant Pal Singh, Amity School of Applied

Sciences, Amity University Uttar Pradesh, Lucknow, santpal.ydic.oel@gmail.com

Second Author - Asita Kulshreshtha, Amity School of Applied

Sciences, Amity University Uttar Pradesh, Lucknow,

akulshreshtha@amity.edu

Third Author - Anoop Kumar Srivastava, Dept. of Radiation

Oncology, Dr. RML Institute of Medical Sciences, Lucknow, dranooprmlims@gmail.com

Fourth Author - Sumit Kumar Srivastava, Amity School of Applied Sciences, Amity University Uttar Pradesh, Lucknow, sumit.astro.physics@gmail.com 